Notas de investigación 



\title{
Repertorios discursivos sobre la corrupción política en España: aproximación inicial
}

\author{
Ariadna Rodríguez-Teijeiro, Laura Román-Masedo \\ UNIVERSIDADE DA CORUÑA \\ ariadna@udc.es - laura.roman@udc.es
}

\begin{abstract}
Resumen: Esta nota de investigación presenta los resultados de un trabajo novedoso en el estudio de la percepción social de la corrupción en España. Las fuentes habituales para realizar este tipo de análisis proceden de estudios cuantitativos que miden la percepción de la corrupción, bien entre expertos (como es el caso del bien conocido Índice de Percepción de la Corrupción de Transparencia Internacional), bien entre la población en general (como en el caso de los Barómetros del Centro de Investigaciones Sociológicas en España o de los Eurobarómetros publicados por la Comisión Europea). En nuestro caso, y partiendo de la concepción de la corrupción política como un constructo social, se ha optado por el análisis del discurso de los participantes en varios grupos de discusión y focus groups realizados para una investigación llevada a cabo en la Comunidad Autónoma de Galicia durante el año 2015. Así, este trabajo se enmarca en lo que los especialistas denominan "tercera generación" de herramientas metodológicas para medir la corrupción, y sus resultados dan cuenta de la riqueza de matices que las técnicas cualitativas aportan al conocimiento y análisis de este fenómeno.
\end{abstract}

Palabras clave: percepción social, corrupción, repertorios discursivos, España.

Abstract: This research note presents the results of an innovative work in the study of the social perception of corruption in Spain. The usual sources for this type of analysis come from quantitative studies that measure the perception of corruption, either among experts (such as the well-known Transparency International Corruption Perception Index), or among the general population (as in the case of the Barometers of the Center for Sociological Research in Spain, or the Eurobarometers published by the European Commission). In our case, and starting from the conception of political corruption as a social construct, we opted for the analysis of the discourse of the participants in various discussion groups and focus groups conducted for a research carried out in the Autonomous Community of Galicia during the year 2015. Therefore, this work belongs to what specialists call "the third generation" of methodological tools to measure corruption, and its results show the richness of nuances that qualitative techniques contribute to the knowledge and analysis of this phenomenon.

Key words: social perception, corruption, discursive repertoires, Spain. 


\section{Introducción}

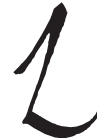

ejos de ser un asunto resuelto, el problema de la corrupción política sigue siendo preocupación principal para una buena parte de gobiernos, organizaciones internacionales y organizaciones no gubernamentales a lo largo y ancho del planeta ${ }^{1}$. Producto de las actividades de este tipo de instituciones son miles las leyes, recomendaciones, evaluaciones y toda suerte de documentos que tratan de contrarrestar, en la medida de lo posible, los efectos perversos de la corrupción ${ }^{2}$, tanto en las democracias consolidadas, como en los países cuyos regímenes políticos están en vías de serlo. Desde el punto de vista de las contribuciones académicas al análisis del fenómeno, su profusión en los últimos años tampoco es en absoluto desdeñable y uno de los principales temas de investigación sigue girando en torno al debate sobre la fiabilidad de los métodos para medir la corrupción. En términos generales, el punto de partida en dicho debate es la constatación de la dificultad para medir directamente la corrupción (Tanzi, 2002:38; Rohwer, 2009:42; Lambsdorff, 2016:82), de manera que hay que acudir a fórmulas indirectas de medición que lo que evalúan, en realidad, son las percepciones de ciudadanos y expertos sobre esta cuestión ${ }^{3}$.

En el contexto anterior, el objetivo de esta nota de investigación es presentar los resultados de un análisis exploratorio que persigue llenar un vacío en el análisis de la percepción social de la corrupción en España. A diferencia de los trabajos mejor conocidos sobre este asunto, nuestra estrategia de investigación ha pasado por la utilización de una técnica poco habitual para analizar este fenómeno como son los grupos de discusión y los focus groups ${ }^{4}$. A largo plazo, y contando con resultados de investigaciones posteriores, de lo que se trataría es de encontrar patrones en los repertorios discursivos de los españoles sobre la corrupción política. Consideramos, por tanto, que este trabajo inaugura una línea de investigación que necesitará de mayor cantidad de material empírico en el futuro. Nos animan en este empeño, tanto los resultados de esta investigación (por parciales que sean), como el exhorto de algunos especialistas

1. En la página web del "Grupo de Estados contra la corrupción" (GRECO) del Consejo de Europa se da buena cuenta de este hecho.

2. Entre dichos efectos perversos puede destacarse el interés suscitado por la relación entre la corrupción y la desafección política (Jimenez, 2017; Torcal, 2014; Della Porta: 2000) y la confianza social y política (Güemes, 2018). Ambas relaciones afectan directamente a los elementos esenciales de la cultura política en una democracia. Sin embargo, los daños de la corrupción política para la democracia pueden afectar potencialmente a cualquier ámbito que tenga alguna responsabilidad en la distribución de bienes públicos (Warren, 2004). Igualmente, las consecuencias perversas de la corrupción sobre la economía han sido objeto de análisis por numerosos autores (Abed y Gupta, 2002; Kaufmann, 2004; Kaufman y Wei, 1999; Tanzi y Davoodi, 1997; Wei, 2000; Mauro, 1995 y 1997 ; Gray y Kaufman, 1998).

3. Algunos autores van aún más lejos cuando afirman que "if corruption could be measured, it could be probably eliminated. In fact, conceptually it is not even clear what one would want to measure" (Tanzi, 2002:38).

4. Para España el único trabajo que conocemos es un estudio cualitativo del CIS (Estudio 2863) publicado en 2011. 
en el tema: "es necesario acudir a evaluaciones más en detalle si se quiere tener información útil para combatir este problema” (Villoria y Jiménez, 2012a:13).

\section{1. ¿Cómo se mide la corrupción?}

Dada su calidad de "actividad delictiva” y "práctica social oculta” (Jiménez y Villoria, 2009:169; Heindrich y Hodess:19), medir la corrupción política no es tarea sencilla, aunque las dificultades quizás comiencen por la falta de consenso sobre el significado del propio término "corrupción" (Rohwer, 2009). Otros obstáculos se desprenden de la complejidad del propio fenómeno que pretende medirse puesto que, a diferencia de otros asuntos que entran en la agenda pública, a la construcción social de este problema contribuyen un número de actores y factores que confieren al tema el carácter multidisciplinar que lo define de manera integral. Así, a las distintas cuestiones que se plantean alrededor del "problema corrupción" pueden responder autoridades procedentes de las especialidades académicas más dispares y también individuos oriundos del proceloso universo de la política, es decir, que en este caso el problema se construye con las aportaciones de aquellos que la interpretan como un objeto de estudio, con las de aquellos que manipulan los datos y los casos, e incluso con las de aquellos que son potencial o ciertamente corruptos. Filósofos, juristas, científicos sociales, economistas, periodistas, políticos, partidos políticos, grupos de presión... casi todo el mundo tiene algo que decir sobre qué es la corrupción y cuánta corrupción hay. De manera que, así las cosas, la tarea de medir cuantitativamente la corrupción se revela misión casi imposible. ¿Medir qué? ¿Cuánta corrupción exactamente de qué tipo ( grand o petty, moral o legal, ocasional o endémica, individual u organizada)? ¿Cuántos casos? ¿Cuantos políticos implicados? ¿Cuántos funcionarios? ¿Cuántos condenados? ¿Cuántos en prisión preventiva? ¿Cuántas diligencias abiertas? ¿Cuántas cerradas? ¿Cuántas portadas de periódico? ¿Cuánto dinero del erario público desviado?

Sin embargo, las preguntas anteriores no han de conducir a un callejón sin salida en la tarea de medir cuantitativamente la corrupción en un país ${ }^{5}$. Es obvio que existen datos objetivos para hacerlo, principalmente si lo que queremos saber es el número de actuaciones judiciales o policiales relacionadas con la corrupción (casos abiertos, investigaciones en marcha, sentencias firmes, número de acusaciones o de condenas, etc.). El problema es que ni siquiera las estadísticas oficiales proceden de una misma fuente, lo que dificulta de nuevo el cómputo de los totales. En el caso de España, esos datos proceden de distintas instancias como la Fiscalía General del Estado, la propia

5. El asunto se complica si lo que se pretende es hacer comparaciones internacionales, puesto que los datos procedentes de fuentes policiales o judiciales pueden tipificar como delito actividades distintas en cada país. Otros indicadores "objetivos" como la existencia o no de leyes y autoridades anti-corrupción, o los fondos que reciben las agencias gubernamentales responsables de este asunto se refieren, más bien, a los tipos de políticas anticorrupción. 
Fiscalía Anticorrupción, la Unidad Central Operativa (UCO) de la Guardia Civil, la Unidad contra los Delitos Económicos y Financieros (UDEF) del Cuerpo Nacional de Policía o el propio Ministerio del Interior (Villoria y Jiménez, 2012a:16 y ss.). Así, las dificultades para componer los totales con datos procedentes de distintas fuentes que además utilizan sus propios métodos de cálculo, conduce irremediablemente a manejar datos "objetivos" parciales que sólo pueden ofrecer una imagen incompleta de cuánta corrupción hay en España. Por último, los obstáculos para responder la pregunta "a partir de qué número de casos debe dispararse la alarma, tomándose la lucha anticorrupción como prioridad nacional y empezando a implantar medidas drásticas" (Villoria y Jiménez, 2012a:21 y ss.), no hacen sino incidir en el dudoso valor científico (que no judicial o político) de los métodos que pretenden despejar la duda de cuánta corrupción hay en España.

Tanto para España como a nivel internacional y dadas las dificultades anteriores, la mayoría de las mediciones que se manejan no son en realidad las que se refieren a "cuánta corrupción hay", sino las relativas a "cuánta percepción del grado de corrupción hay". En este sentido, dos son las herramientas de uso más habitual para medir el fenómeno de la corrupción: las encuestas de percepción (a expertos, ejecutivos, empresarios, etc. o a la ciudadanía en general ${ }^{6}$ ) y las encuestas de victimización.

Entre las encuestas de percepción a expertos mejor conocidas se encuentran las de Tranparencia Internacional, organización que elabora periódicamente su Índice de Percepción de la Corrupción (IPC), y las del Banco Mundial a través del proyecto "Worldwide Governance Indicators" (WGI), en las que se incluye como una de las dimensiones de gobernanza el control de la corrupción. En ambos casos se trata de índices formados por indicadores compuestos basados en fuentes de datos internacionales que miden la percepción de la corrupción a través de encuestas de opinión realizadas a expertos, analistas y personas del mundo de los negocios. Para elaborar el IPC del año 2017 se utilizaron 13 fuentes de datos distintas ${ }^{7}$, mientras que en el caso del WGI, el número más reciente ${ }^{8}$ de fuentes de datos para la dimensión "control de la corrupción" asciende a $32^{9}$.

Ninguno de ambos índices se ha librado de recibir críticas en los últimos años (Villoria y Jiménez, 2012a y 2012b; Treisman, 2007; Andersson y Heywood, 2009; Knack, 2007; Arndt y Oman, 2006; Sampford et al., 2016), aunque entendemos que sus déficits pueden ser compensados al menos por las siguientes razones: $1^{\circ}$ ) conocer lo que la gente piensa y siente sobre el fenómeno de la corrupción y cómo lo evalúa es científicamente tan importante como conocer otras actitudes de la ciudadanía hacia

\footnotetext{
6. Como son los Eurobarómetros publicados por la Comisión Europea o los barómetros del CIS en España.

7. Toda la información está disponible en la página web de Transparencia Internacional.

8. Worldwide Governance Indicators (http://info.worldbank.org/governance/wgi/index.aspx\#home, consultado el 9 de julio de 2018).

9. Una descripción detallada de ambos índices puede consultarse en Rohwer (2009), Malito (2014) y Lucic et al. (2016), entre otros.
} 
cuestiones tales como la legitimidad de un régimen político o el papel del individuo en la política; $2^{\circ}$ ) desde el momento en que el problema de la corrupción política es una construcción social, es fundamental conocer lo que los ciudadanos (expertos en particular y ciudadanos en general) tienen que aportar a la explicación del fenómeno; y $3^{\circ}$ ) en definitiva, y como sostiene Leslie Holmes, "perception is a form of reality" (Holmes, 2015:41).

El segundo de los métodos más utilizados para medir la percepción de la corrupción son los "experiential surveys" o encuestas de victimización. En este caso ya no se pregunta a los ciudadanos sobre sus percepciones del fenómeno de la corrupción (actitudes), sino sobre sus experiencias concretas en actos que son claramente corruptos (como, por ejemplo, pagar un soborno a un funcionario público). La propia Transparencia Internacional lleva a cabo este tipo de encuestas desde el año 2003 en su Global Corruption Barometer que, a diferencia del IPC, utiliza datos propios encargando el trabajo de campo a institutos de opinión en los países en los que se lleva a cabo la investigación. Las entrevistas personales y telefónicas son las técnicas normalmente utilizadas.

El hecho de que los anteriores sean los métodos habituales para medir el nivel de percepción de la corrupción no significa que sean los únicos. Leslie Holmes (2015) recoge otras técnicas de naturaleza claramente minoritaria que también se vienen utilizando para medir el grado de corrupción o percepción de la misma en distintos contextos, si bien de manera excepcional: $1^{\circ}$ ) Tracking surveys ${ }^{10} .2^{\circ}$ ) Método Delphi. $3^{\circ}$ ) Entrevistas. $4^{\circ}$ ) Análisis de contenido de los medios de comunicación. $5^{\circ}$ ) Experimentos de campo y laboratorio. $5^{\circ}$ ) Enfoque proxy $y^{11} .6^{\circ}$ ) Grupos de discusión. Las razones para utilizar o no estos métodos son diversas y no es objetivo de esta nota de investigación entrar en este debate. Sin embargo, son cada vez más los especialistas que apuntan a la existencia de una tercera generación de instrumentos de medición en la que lo recomendable sería la utilización de estos métodos junto con las más tradicionales en orden "to provide a comprehensive and nuanced assessment of a corruption issue in an specific context" (Heinrich y Hodess, 2011:26). En el desplazamiento del interés por los datos comparados a nivel internacional hacia los centrados en la detección de riesgos a nivel local, los grupos de discusión y los focus groups se antojan técnicas de primera importancia.

10. Estas encuestas de seguimiento son utilizadas principalmente por el Banco Mundial con el objeto de conocer el destino de los fondos provistos por esta institución en países en vías de desarrollo (fondos con alguna frecuencia desviados de sus propósitos originales). Se trata básicamente de averiguar cómo se invierten dichos fondos en actividades de carácter social (educación, salud, etc...) que no sólo suministran estadísticas sobre el uso del dinero del Banco Mundial en dichos países, sino que además arrojan buenos resultados tanto para medir, como para "corregir" los casos de corrupción.

11. Utilizado por la organización Global Integrity, se trata de un modo indirecto de medición de los niveles de corrupción en un determinado país mediante el análisis de las medidas que en él se toman (y se implementan) en la lucha contra este fenómeno. 


\section{Metodología y material empírico}

Como ya hemos adelantado, la aproximación metodológica al objeto de estudio de este trabajo parte de la concepción de la corrupción como un constructo social (Tänzler et al., 2012) y, por tanto, entiende que sus significados dependerán de los contextos socio-culturales en los que la corrupción se produce ${ }^{12}$. Así, "it is important in this perspective to consider how the concept is articulated in the framework of different corruption discourses" (Tänzler et al., 2012:21). De esta forma, la percepción de los individuos sobre el fenómeno adquiere principalmente una dimensión cualitativa, puesto que no se trata tanto de saber cuánta corrupción percibe la ciudadanía cuanto de cómo la percibe. En este sentido, conocer el relato de las representaciones propias se hace imprescindible. En el caso de España, el recurso a este tipo de análisis se hace particularmente interesante puesto que la investigación empírica sobre la corrupción política en nuestro país es ciertamente escasa (Jiménez y Villoria, 2009:169). De hecho, ni siquiera el CIS "se ha preocupado hasta el momento de construir una sólida serie histórica sobre la percepción de la corrupción en España" (Jiménez, 2017:2) ${ }^{13}$. Pero además, la introducción de técnicas cualitativas para estudiar el fenómeno de la corrupción incorpora claramente nuestra investigación a la tercera generación de herramientas metodológicas de medición, caracterizada por estrategias de análisis más participativas y por la necesidad de recurrir a la triangulación de técnicas en orden a perfeccionar los grados de fiabilidad de los resultados (Heinrich y Hodess, 2011).

En el contexto anterior, los discursos sobre la naturaleza del fenómeno de la corrupción en España han sido el material de análisis de nuestra investigación. Desde el punto de vista metodológico, la cuestión se ha abordado mediante el análisis del discurso producido en 4 Grupos de Discusión (en adelante GD) y 4 Focus Group (en adelante FG) realizados en distintos puntos de la Comunidad Autónoma gallega a lo largo del año 2015 y cuyas características, criterios de definición y perfil diseñado para los participantes se recogen en las Tablas 1 y $2 .{ }^{14}$

12. Por esto no se entiende que existan formas de corrupción "típicas" de tal o cual cultura, sino que es posible determinar en cada cultura su propio "corruption complex" (Olivier de Sardan, 1999).

13. La escasez de estadísticas e indicadores y la ausencia de estudios cualitativos que aborden este asunto dejó sin explicación, por ejemplo, algunas contradicciones que Jiménez y Villoría encontraron hace algunos años entre los datos del IPC de Transparencia Internacional para España y los sondeos del CIS, o la distorsión entre los datos sobre percepción y preocupación que ponían de manifiesto esos mismos sondeos (Jiménez y Villoria, 2009). Si bien es cierto que la situación en la actualidad ha cambiado (Jiménez, 2017), es obvio que cuestiones como las anteriores necesitan de evaluaciones más profundas para ser abordadas.

14. Dicho material empírico procede de la investigación "Análisis sociológico de Galicia” realizada a través de la Fundación Universidad de A Coruña en el año 2015 (Ref. NV06915). 
Tabla 1

Número de participantes, criterios de definición y perfil diseñado en los GD

\begin{tabular}{|c|c|c|c|c|}
\hline & GD 1 & GD 2 & GD 3 & GD 4 \\
\hline & $\begin{array}{c}\text { Monforte de Lemos } \\
\text { (Lugo) }\end{array}$ & $\begin{array}{l}\text { Vimianzo } \\
\text { (A Coruña) }\end{array}$ & $\begin{array}{l}\text { Lugo } \\
\text { (Lugo) }\end{array}$ & $\begin{array}{l}\text { Ribadavia } \\
\text { (Orense) }\end{array}$ \\
\hline № participantes & 7 & 7 & 7 & 7 \\
\hline $\begin{array}{l}\text { Criterios de } \\
\text { definición }\end{array}$ & $\begin{array}{l}\text { Residencia en el } \\
\text { municipio y perfil } \\
\text { sociodemográfico. }\end{array}$ & $\begin{array}{l}\text { Residencia en el } \\
\text { municipio y perfil } \\
\text { sociodemográfico. }\end{array}$ & $\begin{array}{l}\text { Residencia en el } \\
\text { municipio y perfil } \\
\text { sociodemográfico. }\end{array}$ & $\begin{array}{l}\text { Residencia en el } \\
\text { municipio y perfil } \\
\text { sociodemográfico. }\end{array}$ \\
\hline $\begin{array}{l}\text { Perfil diseñado para } \\
\text { los participantes }\end{array}$ & $\begin{array}{l}\text { Población de } \\
50 \text { a } 70 \text { años, } \\
\text { hombres y mujeres } \\
\text { en proporción } \\
\text { similar, nivel de } \\
\text { estudios primarios, } \\
\text { empleados, } \\
\text { jubilados y } \\
\text { pensionistas y nivel } \\
\text { de ingresos bajos y } \\
\text { medios. }\end{array}$ & $\begin{array}{l}\text { Población de } 20 \text { a } \\
40 \text { años, hombres } \\
\text { y mujeres en } \\
\text { proporción similar, } \\
\text { nivel de estudios } \\
\text { primarios y medios, } \\
\text { empleados y } \\
\text { parados y nivel de } \\
\text { ingresos bajos y } \\
\text { medios. }\end{array}$ & $\begin{array}{l}\text { Población de } 30 \text { a } \\
50 \text { años, hombres } \\
\text { y mujeres en } \\
\text { proporción similar, } \\
\text { nivel de estudios } \\
\text { medios y altos, } \\
\text { empleados y nivel } \\
\text { de ingresos medios } \\
\text { y altos. }\end{array}$ & $\begin{array}{l}\text { Población de } 20 \text { a } \\
40 \text { años, hombres } \\
\text { y mujeres en } \\
\text { proporción similar, } \\
\text { nivel de estudios } \\
\text { primarios y medios, } \\
\text { empleados y } \\
\text { parados, nivel de } \\
\text { ingresos bajos y } \\
\text { medios. }\end{array}$ \\
\hline
\end{tabular}

Fuente: Elaboración propia.

Tabla 2

Número de participantes, criterios de definición y perfil diseñado en los FG

\begin{tabular}{|c|c|c|c|c|}
\hline & FG 1 & FG 2 & FG 3 & FG 4 \\
\hline & $\begin{array}{c}\text { Ferrol } \\
\text { (A Coruña) }\end{array}$ & $\begin{array}{l}\text { Villalba } \\
\text { (Lugo) }\end{array}$ & $\begin{array}{l}\text { Noia } \\
\text { (A Coruña) }\end{array}$ & $\begin{array}{c}\text { Vigo } \\
\text { (Pontevedra) }\end{array}$ \\
\hline № participantes & 7 & 7 & 7 & 7 \\
\hline $\begin{array}{l}\text { Criterios de defi- } \\
\text { nición }\end{array}$ & $\begin{array}{l}\text { Residencia en el } \\
\text { municipio y perfil } \\
\text { sociodemográfico. }\end{array}$ & $\begin{array}{l}\text { Residencia en el } \\
\text { municipio y perfil } \\
\text { sociodemográfico. }\end{array}$ & $\begin{array}{l}\text { Residencia en el } \\
\text { municipio y perfil } \\
\text { sociodemográfico. }\end{array}$ & $\begin{array}{l}\text { Residencia en el } \\
\text { municipio y perfil } \\
\text { sociodemográfico. }\end{array}$ \\
\hline $\begin{array}{l}\text { Perfil diseñado para } \\
\text { los participantes }\end{array}$ & $\begin{array}{l}\text { Población de } 30 \text { a } \\
50 \text { años, hombres y } \\
\text { mujeres en propor- } \\
\text { ción similar, nivel } \\
\text { de estudios medios } \\
\text { y superiores, } \\
\text { empleados y nivel } \\
\text { de ingresos medios } \\
\text { y altos. }\end{array}$ & $\begin{array}{l}\text { Población de } 50 \text { a } \\
70 \text { años, hombres y } \\
\text { mujeres en propor- } \\
\text { ción similar, nivel de } \\
\text { estudios primarios, } \\
\text { empleados, jubila- } \\
\text { dos y pensionistas } \\
\text { y nivel de ingresos } \\
\text { bajos y medios. }\end{array}$ & $\begin{array}{l}\text { Población de } 20 \text { a } \\
40 \text { años, hombres y } \\
\text { mujeres en propor- } \\
\text { ción similar, nivel de } \\
\text { estudios primarios y } \\
\text { medios, empleados } \\
\text { y parados y nivel } \\
\text { de ingresos bajos y } \\
\text { medios. }\end{array}$ & $\begin{array}{l}\text { Población de } 30 \text { a } \\
50 \text { años, hombres y } \\
\text { mujeres en propor- } \\
\text { ción similar, nivel } \\
\text { de estudios medios } \\
\text { y superiores, em- } \\
\text { pleados y parados } \\
\text { y nivel de ingresos } \\
\text { medios y altos. }\end{array}$ \\
\hline
\end{tabular}


Por lo que se refiere al modo de abordar el análisis del discurso de los diferentes participantes en los GD y FG, hemos seguido dos fases diferenciadas: (1) una primera consistente en la descomposición analítica del texto en unidades básicas de significación, es decir, la detección y análisis de las diferentes opiniones vertidas en relación a las cuestiones específicas relativas a nuestro objeto de investigación; y (2) un análisis más general de la dinámica del texto, es decir, del sentido global del mismo. Así, el resultado de dicho análisis nos ha proporcionado: (1) la determinación de los distintos «repertorios discursivos» existentes en el corpus del texto de investigación; (2) la delimitación de las «configuraciones narrativas» entendidas como hilos conductores que vertebran el discurso y dan estructura a las diferentes opiniones o posiciones discursivas existentes dentro del grupo; y (3) el planteamiento de los distintos «espacios semánticos» (Conde, 2009) como el análisis y estructuración del concepto del que se habla y de cómo se organizan las relaciones y posibles asociaciones entre los elementos del discurso. En definitiva, hemos tratado de detectar los ejes multidimensionales que estructuran el contenido de los textos discursivos, la trama interna de lo que se dice y de lo que se pretende decir, y de buscar las consideraciones u opiniones organizadoras del debate que se establece en los distintos GD y FG realizados.

Podemos adelantar que, en base a los objetivos de nuestra investigación, hemos descubierto diferentes posiciones y repertorios discursivos relativos al fenómeno de la corrupción política que podemos agrupar de la siguiente manera: (1) la percepción de la corrupción en términos generales; (2) los posibles factores causantes de los diferentes casos de corrupción; y (3) la identificación de posibles consecuencias e implicaciones derivadas de los mismos.

Terminamos este apartado metodológico señalando que el material empírico descrito más arriba es producto de una investigación que no estaba originalmente pensada para analizar la percepción social de la corrupción. La riqueza de los datos que se obtuvieron en los GD y FG respecto a este asunto pusieron de manifiesto hasta qué punto el fenómeno de la corrupción se ha situado en el subconsciente colectivo, se mantiene como una cuestión muy presente en la agenda social (y mediática) ${ }^{15} \mathrm{y}$ necesita de análisis cualitativos más profundos. La espontaneidad de los participantes a la hora de abordar una cuestión sobre la que no se les estaba preguntando da buena cuenta de esta necesidad.

15. En efecto, Ramió (2016) en su análisis sobre las causas más relevantes de la corrupción en España plantea el alcance transversal de la misma que la vincula con el tejido empresarial, sindicatos y medios de comunicación, y no solamente con los partidos políticos y sus dirigentes. 


\section{Análisis y resultados de la investigación}

Basándonos en los discursos predominantes en los GD y FG, podemos afirmar que nuestro tema de investigación constituye una problemática de naturaleza multidimensional. En concreto, se constata la vinculación de la corrupción en términos generales con aspectos relativos a otros espacios o realidades como son: a) el ámbito económico (aludiendo a cuestiones tales como la crisis económica y/o la gestión de la misma); b) el ámbito político (y en concreto con el ejercicio y el concepto de poder, así como con el funcionamiento interno de los partidos políticos); c) el ámbito institucional (señalando el concepto de democracia y su relación con el posible cambio político); y por último, d) el ámbito social (donde se ubica de manera específica la percepción de la población hacia la corrupción y hacia la política en general, así como la distancia y desafección hacia la misma).

\section{Figura 1}

Dimensiones vinculadas con la corrupción contenidas en el discurso emitido en los GD y FG

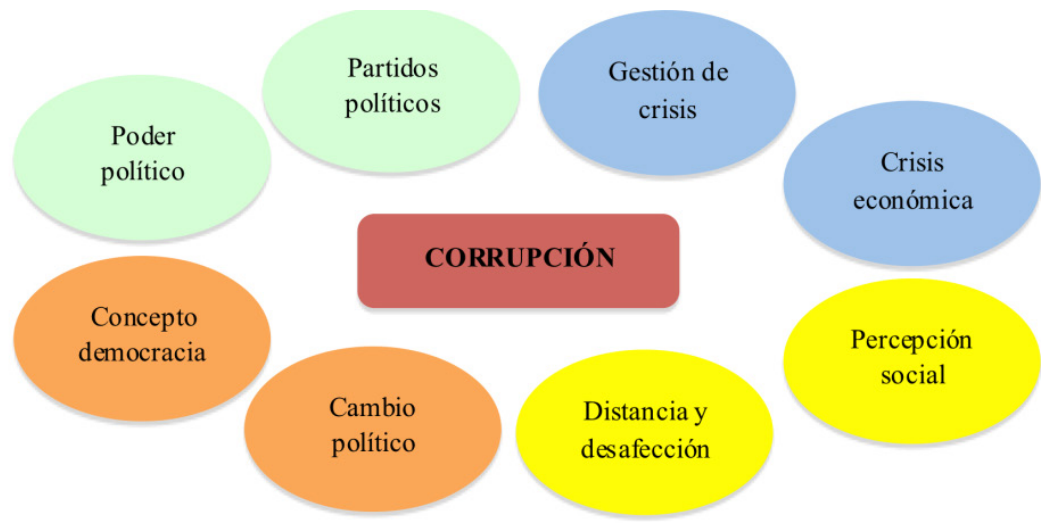

Fuente: Elaboración propia a partir de los discursos contenidos en los GD y FG realizados.

\section{1. Ámbito económico: crisis económica y corrupción}

Como se desprende de los siguientes repertorios discursivos, los participantes en los GD y FG vinculan la gestión de la crisis económica y la gestión de los casos de corrupción, percibiéndolos como dos caras de la misma moneda.

Inicialmente la corrupción se asocia de manera directa con el Partido Popular y se equipara la gestión de la crisis con la gestión de los casos de corrupción. Así, se 
alude a ambas en los mismos términos de mala gestión, tanto por parte del presidente de un partido, como por parte del Presidente del Gobierno, lo cual muestra cierta confusión en cuanto a los roles de presidente del partido/Presidente del Gobierno, así como sobre la asignación de distintos niveles de responsabilidad en la gestión de la crisis económica y de los casos de corrupción. Dicha asociación entre ambos roles equipara la "mala" gestión de la crisis económica con la "deficiente" gestión de la corrupción, ambas entendidas como responsabilidad de un mismo partido. Los participantes se muestran firmes y con una actitud dura a la hora de criticar lo que consideran como falta de respuesta y de contundencia por parte del PP ante los distintos casos de corrupción. No es así -o al menos no en la misma medida- con los casos de corrupción vinculados a otros partidos y, en concreto, con aquellos protagonizados por miembros o dirigentes del PSOE. En este sentido, parece manifestarse en el discurso producido por parte de determinados sectores de participantes en los GD y FG, que se infringe un mayor castigo al PP por los casos de corrupción debido al hecho de ser el partido que estaba en el Gobierno.

En este sentido, pretendíamos plantear diferentes ejes discursivos y espacios semánticos (en adelante ES) en los que se condensan las principales percepciones respecto a la cuestión de la corrupción política y la gestión de la misma, así como su vinculación con la crisis económica.

Gráfico 1

Representación de los ejes vertebradores del discurso contenido en el corpus del texto de los GD y FG

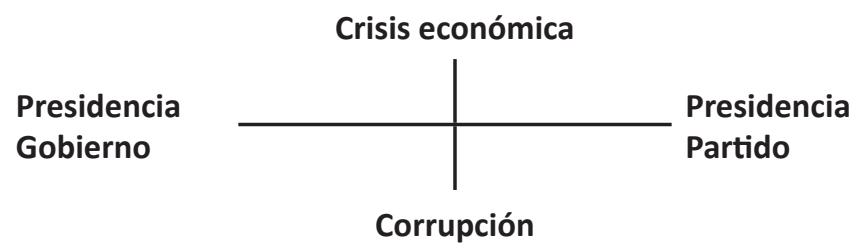

Fuente: Elaboración propia.

Así, los ejes discursivos que vertebran el corpus de investigación y las configuraciones narrativas relativas a este ámbito serían los siguientes:

Eje 1: Un eje discursivo correspondiente a los roles de presidente de un partido y de Presidente del Gobierno.

Eje 2: Un segundo eje discursivo que alude al concepto de gestión y que oscila desde la gestión de la crisis económica hasta la gestión de la corrupción. En este caso, 
podemos decir que la corrupción invade los cuatro espacios semánticos resultantes del cruce de ambos ejes discursivos, no siendo posible a partir del material empírico disponible llegar a establecer espacios semánticos diferenciados ${ }^{16}$.

Los cruces de configuraciones narrativas se ponen de manifiesto cuando:

a Los participantes plantean sus discursos sobre sobre crisis económica y señalan como posibles causas o culpables de la misma, en primer lugar, a "los políticos avariciosos que despilfarran y se enriquecen"; y a continuación, a los numerosos casos de corrupción existentes: "las arcas están vacías por los que roban" (GD No 2) (señalando también a los bancos como responsables de la crisis, en tercer lugar).

b Un segundo cruce de configuraciones narrativas entre el Eje 1 de crisiscorrupción se combina con el Eje vertebrador 2 correspondiente a los que se señalan como posibles culpables de la situación de crisis económica, a saber, los políticos y la corrupción.

\section{2. Ámbito político: poder, partidos políticos y corrupción}

La corrupción se percibe asociada, en segundo lugar, a conceptos de naturaleza claramente política, como son el poder y su ejercicio y los partidos políticos.

Respecto al primero de ellos, un sector importante de los participantes manifiesta de manera más o menos explícita la idea de poder = corrupción, es decir, la consideración de que los políticos "cuando llegan al poder se corrompen" (GD № 3 ). Los participantes entienden, por tanto, que los políticos modifican su actitud respecto a determinadas prácticas o acciones poco lícitas aunque mantengan intacto su discurso respecto a las mismas. En este caso podemos decir que se vincula casi de manera inmediata el ejercicio del poder con las prácticas corruptas.

Otro aspecto detectado en el discurso de los participantes en los GD y FG es la idea de que los políticos están "todos metidos en el mismo saco de la corrupción” (FG No 2), idea planteada de manera transversal, es decir, que incluye a todos los políticos por igual independientemente del partido al que pertenezcan. Además, se asocia toda actividad política que tenga que ver con la gestión de un partido político con la tendencia casi inevitable a la corrupción, lo cual pone de manifiesto cierta contradicción con la identificación de la corrupción con un partido en concreto, como veíamos en el apartado anterior. En este sentido, se defiende de manera casi unánime

16. El material empírico disponible no nos ha permitido llegar a establecer los ES específicos resultantes del cruce de ambos ejes discursivos para este ámbito. Esta es la razón del carácter exploratorio de nuestra investigación, que necesitará de posteriores trabajos para alcanzar mayor profundidad en el análisis de los repertorios discursivos de la población a nivel nacional en relación a esta cuestión. 
la idea de que "no se corta por lo sano con la corrupción porque si no se quedarían sin partidos" (FG No 1).

Asimismo, se denuncia de manera reiterada en distintos grupos y por parte de diferentes perfiles de participantes la permisividad y connivencia que existe dentro de los propios partidos políticos hacia los casos de corrupción interna, entendiendo ésta como aquella generada en el seno de un partido u organización política concreta. Esta percepción llega al punto de que diversos participantes en los GD y FG opinan que los partidos políticos tienen una naturaleza eminentemente delictiva, de forma que la corrupción es entendida como un mal endémico del sistema. Por otro lado, del material empírico analizado se desprende que la imagen de los políticos se encuentra en términos generales desvirtuada debido, en gran parte, al alto grado de corrupción percibida. En este sentido, encontramos cuatro actores "causantes" de esa situación distribuidos en dos Ejes discursivos: Eje 1: Políticos - sistema; Eje 2: Ciudadanos justicia como se puede ver en el siguiente Gráfico 2.

\section{Gráfico 2}

Representación de los ejes vertebradores y de los espacios discursivos contenidos en el corpus del texto de los GD y FG

\begin{tabular}{l|l|l}
\multicolumn{2}{c}{ Ciudadanos } \\
Políticos & PERMISIVIDAD & MAL ENDÉMICO \\
\cline { 2 - 3 } CONNIVENCIA & LEY LAXA
\end{tabular}

Justicia

Fuente: Elaboración propia.

Así, del cruce de ambos ejes vertebradores del discurso contenido en los GD y FG sobre los potenciales culpables de la corrupción y su nivel de afectación resultan cuatro espacios semánticos en los que se condensan diferentes visiones en torno al origen y los posibles factores agravantes de la corrupción.

ES 1: Un primer espacio semántico en el que los ciudadanos señalan y sitúan la corrupción política como un mal endémico de nuestro sistema político.

ES 2: Un segundo ES en el que se señala como culpable del grado de corrupción existente (percibida) a la justicia por aplicar una ley laxa hacia los casos de corrupción juzgados. 
ES 3: Un tercer espacio en el que se sitúa la percepción de que en nuestro país existe cierta connivencia entre los políticos y la justicia.

ES 4: Y por último, un cuarto espacio en el que se ubica un discurso que denuncia la existencia de permisividad por parte de los ciudadanos respecto a las irregularidades, ambiciones y abusos en los que casi siempre, según manifiestan, "caen los políticos tarde o temprano" (GD No 3).

\section{3. Ámbito institucional: democracia, cambio político y corrupción ${ }^{17}$}

En lo relativo al ámbito institucional, los participantes en los GD y FG ponen en relación el concepto de democracia y los procesos de cambio político con la corrupción, vinculando ambos a través de la idea de participación política de la ciudadanía.

En términos generales, el concepto de democracia implícito en el discurso de varios sectores de participantes en los GD y FG defiende la necesidad de una mayor participación de los ciudadanos en la vida política, e incluso su implicación en la gestión al objeto de que dicha participación ayude a "no estafar, a no robar, a mejorar la sanidad..."'. Así, del discurso de los participantes se deduce que la participación política de la ciudadanía podría evitar los casos de corrupción e impedir que accedieran al gobierno miembros de partidos políticos no honrados o deshonestos ${ }^{19}$. Observamos como, nuevamente, se proyecta la corrupción de los partidos a la acción de gobierno.

Por otro lado, la promoción y búsqueda del cambio político por parte de algunos sectores de la población parece tener más que ver con la corrupción que con la crisis económica. La gente está dispuesta a apoyar a los nuevos partidos, incluso "aunque no se sepa qué nos espera" (FG No 3). Este hecho se considera como una de las medidas desesperadas que el individuo puede adoptar ante una situación compleja, de la misma manera que, dicen, "cuando te tienes que ir del país a buscar trabajo o cuando estás desahuciado". Dichos sectores apuntan a que esta actitud no está provocada tanto por el sufrimiento, cuanto por el cansancio de la ciudadanía provocado por el hecho tener siempre a los mismos políticos que "no hacen más que robar". Por tanto, son los casos de corrupción más que la crisis económica los que promueven el mantenimiento de este repertorio discursivo.

17. Véase nota 16.

18. Sin embargo, algunos sectores grupales critican la falta de implicación ciudadana desde la perspectiva crítica de que "es mejor echarles la culpa a los políticos y no hacer nada" (GD № 1).

19. Para un análisis sobre las preferencias de la ciudadanía sobre quién debe tomar las decisiones políticas puede consultarse el artículo de Río, Navarro y Font (2016) citado en la bibliografía. 


\section{4. Ámbito social: desafección política y corrupción}

Terminamos refiriéndonos a las posiciones discursivas y configuraciones narrativas relacionadas con la vinculación que los participantes establecen entre corrupción y desafección política. Para este apartado, hemos identificado dos ejes discursivos y cuatro ES.

Eje 1 de actores: justicia - clase política. Aquí encontramos dos percepciones. En primer lugar, la de la justicia como institución que ha de velar por el adecuado cumplimiento de las leyes así de como dictar sentencias ecuánimes y ajustadas al concepto que de la misma tienen los participantes. Por otro lado, la percepción de la clase política como un concepto difuso un tanto elástico desde el punto de vista de que la población parece no discriminar entre políticos en general, representantes desde el punto de vista institucional, gestores o responsables de un partido.

Eje 2 de acciones: gestión (deficiente) - corrupción. En este eje encontramos, por un lado, la existencia de una mala praxis -gestión deficiente desde el punto de vista institucional- $y$, por el otro, la propia corrupción política.

\section{Gráfico 3}

Representación de los ejes vertebradores y de los espacios discursivos contenidos en el corpus del texto de los GD y FG

\begin{tabular}{ll|l} 
& \multicolumn{2}{c}{ Justicia } \\
Deficiente & NO ECUÁNIME & IMPUNIDAD \\
\cline { 2 - 3 } gestión & DESILUSIÓN & DESCONFIANZA \\
\multicolumn{2}{c}{ Clase política }
\end{tabular}

Fuente: Elaboración propia.

Del cruce entre ambos ejes detectamos la existencia de distintos ES en los que se condensan, a nuestro juicio, los principales aspectos en los que se sustenta la percepción social de la corrupción en España.

ES 1: En este se plantea la falta de ecuanimidad por parte de la justicia a la hora de juzgar y, eventualmente, penar la mala gestión de la corrupción. Se trata de un concepto basado en una mala práctica de la acción y gestión política.

ES 2: El segundo ES se basa en el concepto de impunidad según el cual se entiende que la mala praxis y las prácticas abusivas o corruptas nos son suficiente- 
mente detectadas, juzgadas y penadas, es decir, se considera que "robar sale barato" (FG No 2).

ES 3: Expresa la desilusión del ciudadano hacia la clase política basada en la deficiente gestión de los asuntos públicos.

ES 4: Y, por último, el cuatro ES en el que se señala la desconfianza de la ciudadanía hacia la clase política debido a la gestión poco honesta, ineficaz y delictiva que engloba la corrupción en general.

En los discursos analizados, estos dos últimos aspectos, desilusión y desconfianza, se relacionan directamente con la corrupción política, lo cual resulta coherente con los resultados que arrojan los estudios de opinión en los que los encuestados sitúan la corrupción como uno de los principales problemas de España ${ }^{20}$. La relación entre clase política y corrupción se manifiesta en algunas opiniones que incluso ponen en cuestión los sueldos de los políticos: "reciben sueldos excesivos para lo que hacen..." (GD No 1).

\section{Conclusiones}

A pesar de su naturaleza limitada, los resultados de esta nota de investigación ponen de manifiesto la necesidad de seguir profundizando en el análisis de la corrupción política en España, utilizando técnicas que superen los más tradicionales estudios de opinión. Como se señaló al principio de este trabajo, ya existe una corriente metodológica que demanda el uso de técnicas de investigación más participativas al objeto de mejorar los niveles de fiabilidad de los resultados en este campo de estudio.

Siguiendo la propuesta metodológica anterior, hemos recurrido al análisis del discurso producto de la realización de cuatro GD y cuatro FG. Dicho análisis nos muestra la existencia de los tres conceptos clave que podrían vertebrar la percepción social de la corrupción en España.

$1^{\circ}$ ) La impunidad, que se asocia al hecho de que parece «no haber castigos para el corrupto", la ley es demasiado laxa con los casos de corrupción y la justicia no es ecuánime, es decir, de que no se trata a todos los políticos corruptos por igual ni se juzgan todos los casos por igual -institucional, mediática y jurídicamente.

$2^{\circ}$ ) La desconfianza de los ciudadanos hacia la clase política en general, lo cual apoya los resultados que de manera constante arrojan los barómetros de opinión elaborados por el CIS. Esto es así hasta el punto de que algunas fracciones grupales de los GD y FG consideran a los políticos como prescindibles, apostando por un

20. Nos referimos a los Barómetros de Opinión elaborados por el CIS. 
gobierno de técnicos como alternativa para mejorar la gestión de los asuntos públicos, corrupción incluida.

$3^{\circ}$ ) La desilusión, como resultado de todo lo apuntado anteriormente, especialmente por parte de las generaciones más jóvenes, desilusión que, lejos de lo que pudiera parecer, procede antes de la percepción de la corrupción política que de la propia crisis económica.

\section{Bibliografía}

ANDERSSON, Staffan y HEYWOOD, Paul M. (2009). “The Politics of Perception: Use and Abuse of Transparency International's Approach to Measuring Corruption". Political Studies, 57: 746-767.

ARNDT, Christiane y OMAN, Charles (2006). Uses and Abuses of Governance Indicators. OCDE Development Centre, París.

CENTRO DE INVESTIGACIONES SOCIOLÓGICAS. (2017). Barómetro Diciembre (Estudio no 3199).

CONDE GUTIÉRREZ DEL ÁLAMO, Fernando (2009). Análisis sociológico del sistema de discursos. Madrid: Centro de Investigaciones Sociológicas.

DELLA PORTA, Donatella (2000). "Social Capital, Beliefs in Goverment, and Political Corruption", en Susan J. Pharr y Robert D. Putnam (eds.), Disaffected Democracies. What's Troubling the Trilateral Countries? Princeton: Princeton University Press.

GRAY, Cheryl W. y KAUFMAN, Daniel (1998). "Corruption and Development". Finance and Development, 35: 7-10.

GÜEMES, Cecilia (2018). "La corrupción y la (des)confianza como normas sociales. Cambo de enfoque, nuevas perspectivas". Revista Internacional de Transparencia e Integridad, 6.

HEINDRICH, Finn y HODESS, Robin (2011). "Measuring corruption" en Graycar, Adam y Smith, Russell G. (eds.) (2011). Handbook of Global Research and Practice on Corruption. Cheltenham: Edward Elgar.

HOLMES, Leslie (2015). Corruption. A very short introduction. Oxford: Oxford University Press.

JIMÉNEZ, Fernando y VILLORIA, Manuel (2009). "Percepción social de la corrupción en España". AFDUAM (Anuario de la Facultad de Derecho de la Universidad Autónoma de Madrid), 12: 169-183.

JIMÉNEZ, Fernando (2017). "Los efectos de la corrupción política sobre la desafección y el cambio político en España”. Revista Internacional de Transparencia e Integridad, 5 . 
KAUFMANN, Daniel (2004). "Corruption, governance and security: challenges for the rich countries and the world", Global Competitiveness Report 2004/2005. Washington: Banco Mundial, 83-102.

KAUFMANN, Daniel y WEI, Shang-Jin (1999). "Does 'grease money' speed up the wheels of commerce?”. Washington D.C.: International Monetary Fund, Working Paper 00/64.

KNACK, Stephen (2007). "Measuring Corruption: a Critique of Indicators in Eastern Europe and Central Asia”. Journal of Public Policy, 27 (3): 255-291.

LAMBSDORFF, Johann Graf (2016). "Measuring Corruption. The Validity and Precision of Subjective Indicators (CPI)" en Sampford, Charles; Shacklock, Arthur; Connors, Carmel y Galtung, Fredrik (eds.) (2016). Measuring corruption. Londres: Routledge.

LUCIC, Danilo; GOLUBOVIC, Natasa y DZUNIC, Marija (2016). "Measuring corruption. Key issues, data sources and the most commonly used indicators". Facta Universitatis (Serie: Economics and Organization), 13 (1): 101-115.

MALITO, Debora Valentina (2014). "Measuring Corruption Indicators and Indices". European University Institute/Robert Schuman Centre for Advanced Studies. Working Paper, 13.

MARTÍNEZ ROSÓN, María del Mar (2016). "Yo prefiero al corrupto: el perfil de los ciudadanos que eligen políticos deshonestos pero competentes". Revista Española de Investigaciones Sociológicas, 153: 77-94.

MAURO, Paolo (1995). "Corruption and Growth". The Quarterly Journal of Economics, $110(3), 681-712$.

MAURO, Paolo (1997). “The Effects of Corruption on Growth, Investment and Government Expenditure”. Washington D.C.: International Monetary Fund, Working Paper 96/98.

OLIVIER DE SARDAN, J-P (1999). “A Moral Economy of Corruption in Africa?”. The Journal of Modern African Studies 37(1): 25-52.

RAMIÓ, Carles. (2016). La renovación de la función pública: estrategias para frenar la corrupción política en España. Madrid: Los Libros de la Catarata.

RÍO, Adrián del; NAVARRO, Clemente J. y FONT, Joan (2016). “Ciudadanía, políticos y expertos en la toma de decisiones políticas: la percepción de las cualidades de los actores políticos importan”. Revista Española de Investigaciones Sociológicas, 154: 83-102.

ROHWER, Anja (2009). "Measuring Corruption: A Comparison between the Transparency International's Corruption Perceptions Index and the World Bank's Worldwide Governance Indicators". CESifo DICE Report 7 (3): 42-52. Munich: Ifo Institute for Economic Research. 
SAMPFORD, Charles; SHACKLOCK, Arthur; CONNORS, CARMEL Y GALTUNG, Fredrik (eds.) (2016). Measuring corruption. Londres: Routledge.

TANZI, Vito (2002). "Corruption Around the World: Causes, Consequences, Scope and Cures" en Abed, George T., Gupta, Sanjeev (eds.) (2002). Governance, Corruption \& Economic Performance. Washington D.C.: International Monetary Fund.

TANZI, Vito y DAVOODI, Hamid. (1997). "Corruption, public investment, and growth", Washington D.C.: International Monetary Fund, Working Paper 97/139.

TÄNZLER, Dirk; MARAS, Konstandinos; GIANNAKOPOULOS, Angelos y ROGOWSKI, Ralf. (2012). "The Social Construction of Corruption: Theorethical Reflections” en Tänzler, Dirk; Maras, Konstandinos; Giannakopoulos, Angelos y Rogowski, Ralf. (eds.) (2012). The Social Construction of Corruption in Europe. Farnham: Ashgate.

TORCAL, Mariano (2014). “The Decline of Political Trust in Spain and Portugal: Economic Performance or Political Responsiveness?” American Behavioral Scientist, 58 (12): 1542-1567.

TORRES ALBERO, Cristobal (2015). España 2015. Situación social. Madrid: Centro de Investigaciones Sociológicas.

TREISMAN, Daniel (2007). "What have we learned about the causes of corruption from ten years of cross-national empirical research?". Annual Review of Political Science, 10: 211-244.

VILLORIA, Manuel y JIMÉNEZ, Fernando (2012a). “¿Cuánta corrupción hay en España? Los problemas metodológicos de la medición de la corrupción (20042011)". Revista de Estudios Políticos, 156: 13-47.

VILLORIA, Manuel y JIMÉNEZ, Fernando (2012b). "La corrupción en España (2004-2010): datos, percepción y efectos". Revista Española de Investigaciones Sociológicas, 138: 109-134.

WARREN, Mark E. (2004). "What Does Corruption Mean in a Democracy?". American Journal of Political Science, 18 (2): 328-343.

WEI, Shang-Jin (2000). "How taxing is corruption on international investors". Review of Economics and Statistics, 82 (1): 1-11. 